\title{
The Efficacy of Herbal Supplements and Nutraceuticals for Prevention of Migraine: Can They Help?
}

Kavaljeet Kaur ${ }^{1}$, Vernicia Hernandez ${ }^{1}$, Sari W. Al Hajaj ${ }^{2}$, Ahmed M. Ebrahim ${ }^{2}$, Mirash Razack 3, 4, 5 , Mohamed W. ElSharief ${ }^{6}$, David Dragas ${ }^{7}$

1. Internal Medicine, California Institute of Behavioral Neurosciences \& Psychology, Fairfield, USA 2. Surgery, California Institute of Behavioral Neurosciences \& Psychology, Fairfield, USA 3. Emergency Medicine, California Institute of Behavioral Neurosciences \& Psychology, Fairfield, USA 4. Medicine, Wuhan University, Wuhan, CHN 5. Internal Medicine, $\mathrm{Al}$ Ain Hospital, $\mathrm{Al}$ Ain, ARE 6. Pediatrics and Child Health, California Institute of Behavioral Neurosciences \& Psychology, Fairfield, USA 7. Research, California Institute of Behavioral Neurosciences \& Psychology, Fairfield, USA

Corresponding author: Kavaljeet Kaur, kavaljeet.kaur89@gmail.com

\begin{abstract}
Migraine is a common neurological disorder associated with or without aura. Although the pathophysiology of migraine is not very well understood, pro-inflammatory cytokines and oxidative stress biomarkers are found to be increased in migraine. Multiple studies have been done to see if alternative medicine such as herbal supplements and nutraceuticals are effective in the prevention and treatment of migraine headaches. This review aimed to evaluate the effect of supplements like coenzyme Q10, riboflavin (vitamin B2), feverfew, and magnesium on the frequency, severity, and duration of migraine attacks.
\end{abstract}

We performed a thorough literature search using mainly PubMed. We included studies published in the last 10 years, those conducted among adult human participants 18-65 years of age, and those published in the English language. Based on the articles selected for the final review, we concluded that herbal supplements and nutraceuticals help reduce the frequency of migraine headaches; however, mixed results were seen regarding the severity and duration of headaches. Moreover, there were no concerning side effects with these supplements. Therefore, physicians can suggest herbal supplements to patients who experience adverse effects from pharmaceutical drugs and desire a more natural treatment.

Received 03/01/2021 Review began 04/19/2021 Review ended 05/02/2021 Published 05/06/2021

○ Copyright 2021 Kaur et al. This is an open access article distributed under the terms of the Creative Commons Attribution License CC-BY 4.0., which permits unrestricted use, distribution, and reproduction in any medium, provided the original author and source are credited.
Categories: Family/General Practice, Internal Medicine, Neurology

Keywords: migraine, headache, nutraceuticals, antioxidants, coenzyme q10, herbal supplements, prevention, riboflavin

\section{Introduction And Background}

Migraine is characterized by moderate-to-severe episodic, throbbing, unilateral headaches usually accompanied by nausea, vomiting, photophobia, and phonophobia [1]. According to the Global Burden of Disease report from the World Health Organization, it is the third most prevalent and the sixth most debilitating disease globally. Migraine is three times more common in females than in males with a worldwide prevalence of $14.7 \%$ [2]. Overall, $2 \%$ of the world's population and, on average, one in seven Americans suffer from migraines [3,4].

The pathophysiology of migraine is unclear. Studies with phosphorus magnetic resonance spectroscopy (31P-MRS) show altered energy metabolism in the brain. These abnormalities in energy metabolism seem to have a genetic component and a neurovascular component. Migraine is associated with a mutation in genes coding for metabolic enzymes in both mitochondrial and nuclear DNA [5-7]. The neuronal and vascular dysfunction observed can be explained by impaired oxygen metabolism resulting from mitochondrial dysfunction, oxidative stress, and inflammation resulting in changes in vascular tone $[8,9]$. Migraine is associated with other disorders such as cardiovascular disease, stroke, asthma, epilepsy, allergies, and sleep disorders [10]. It is usually triggered by fasting, skipping meals, stress, and lack of sleep [11,12].

Migraine is a chronic debilitating condition impacting daily life. Proper treatment and prevention can reduce the burden on healthcare and improve the quality of life of patients. Acute attacks are treated with analgesics or triptans. Drugs used for prophylaxis include anti-depressants (amitriptyline), anti-epileptics (topiramate, valproic acid), beta-blockers (propranolol), and calcium-channel blockers.

Recently, the use of herbal supplements and nutraceuticals such as coenzyme Q10, vitamin B2 (riboflavin), feverfew, and magnesium seem to be gaining popularity for the prevention of migraine. One of the reasons for their rising popularity is fewer side effects in comparison to pharmacological drug therapy. With this review article, we intend to explore the effectiveness of nutraceuticals and herbal supplements for the prevention of migraine attacks. We expect that this article will help guide physicians to decide which 


\section{Cureus}

preventive treatment suit a patient better and thus help with future migraine attacks.

\section{Review}

We performed a thorough literature search using PubMed to identify relevant studies. Studies published in the last 10 years, performed among human adult populations 18-65 years of age, and published in the English language were included. The search was done using keywords "migraine," "nutraceuticals," “supplements," “Coenzyme Q10 ubiquinone,” and “prevention and control.”

The initial search yielded 106 articles, but after careful screening, applying inclusion and exclusion criteria, and excluding duplicate articles, 36 were shortlisted for abstract and full-text review. These included randomized controlled trials, observational studies, cohort studies, systematic reviews, and metaanalysis. Animal studies, case reports, and studies on pediatric and adolescent populations were excluded.

A total of 36 studies selected for the final review included two systematic reviews with meta-analysis, four randomized placebo-controlled double-blinded trials, three open-label pilot studies, one observational study, one cohort study, and review articles. Table 1 presents the studies and their findings.

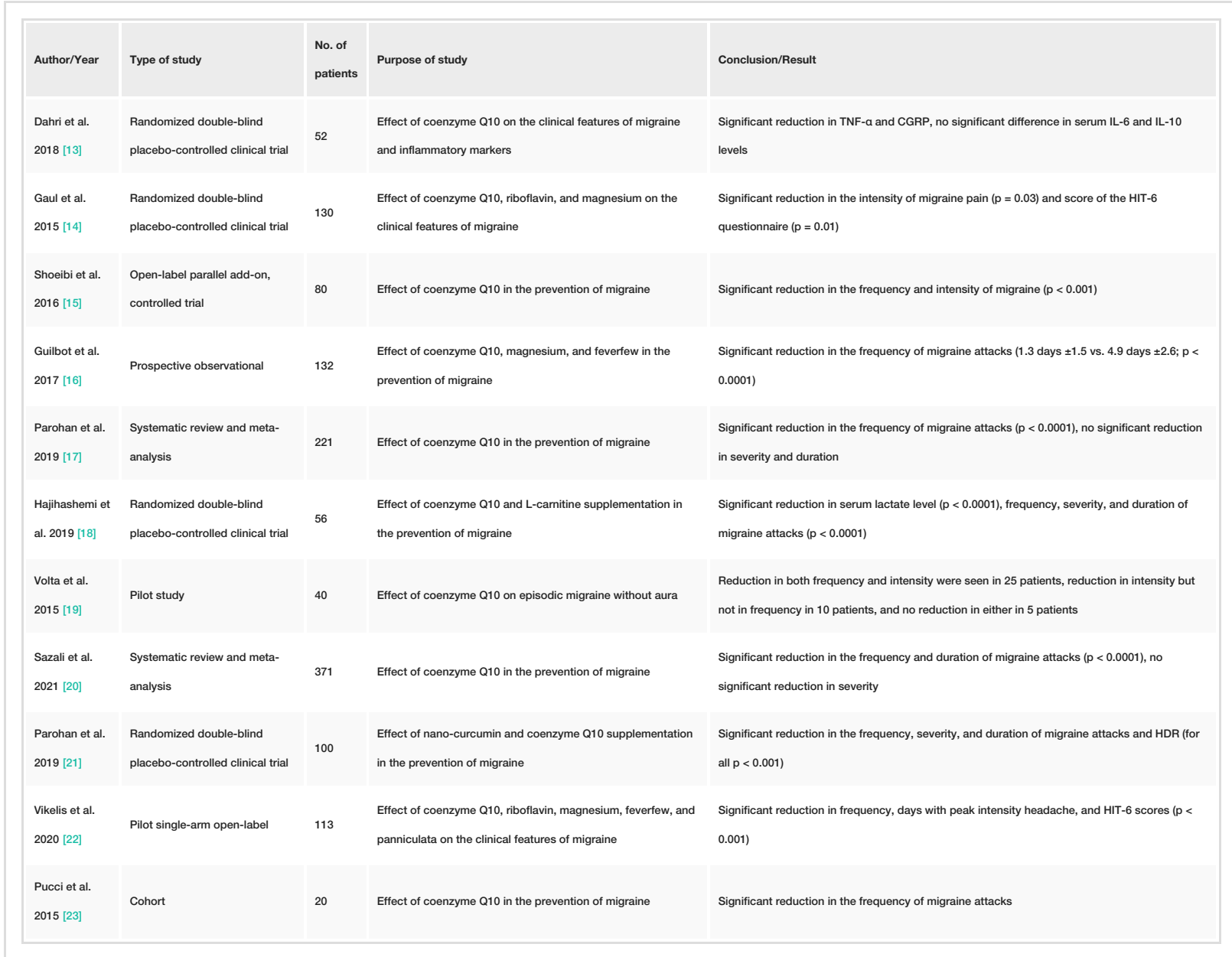

\section{TABLE 1: Effect of nutraceuticals on migraine headache.}

TNF-a: tumor necrosis factor-a; CGRP: calcitonin gene-related peptide; IL-6: interleukin-6; IL-10: interleukin-10; HIT-6: headache impact test; HDR: headache diary results

\section{Discussion}

Migraine is considered to occur due to disturbances in brain homeostasis. The pathophysiology of migraine is unclear but seems to include a genetic component and a neurovascular component. Mutations in chromosome 19 are seen to be linked with familial migraine. Mutations involving Cav2.1 (P/Q)-type voltagegated calcium channel CACNA1A gene are seen in about $50 \%$ cases of familial migraine [24]. One consequence of this mutation may be increased glutamate release. Mutations in the ATP1A2 gene have been identified to be responsible for about $20 \%$ of familial cases. ATP1A2 gene codes for a $\mathrm{Na}+/ \mathrm{K}+$ ATPase. 


\section{Cureus}

Mutation in this gene leads to decreased activity of astrocytic glutamate transporters and eventually buildup of synaptic glutamate. The increased glutamate seems to cause migraine headaches [25]. Figure 1 shows the various triggers and pathophysiology of migraine headache.

Factors

Mechanism
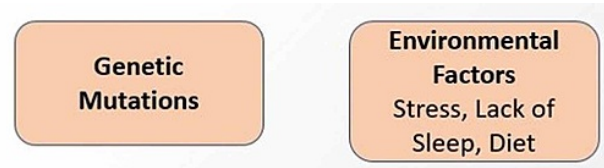

Hormonal Factors
Menstruation
Pregnancy

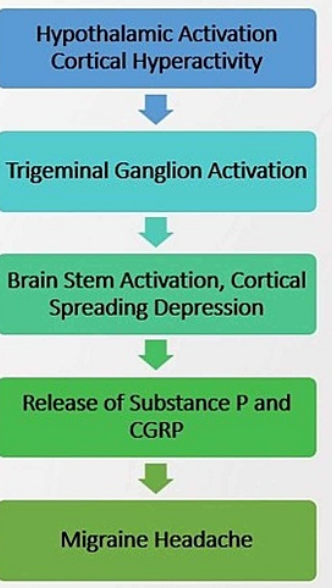

FIGURE 1: Pathophysiology of migraine headache.

CGRP: calcitonin gene-related peptide

The occurrence of migraine is associated with cortical hyperactivity. The trigeminal ganglion is hypothesized to be involved in this hyperactivity [26]. Activation of trigeminal sensory nerve fibers, in turn, causes brainstem activation and induces the release of vasoactive peptides such as substance P and calcitonin gene-related peptide (CGRP). This causes platelet and endothelial activation and, consequently, an increase in nitric oxide synthesis, vasodilation, leakage of blood vessels, and degranulation of mast cells [27]. This further activates sensory trigeminal fibers, increasing the release of substance P and CGRP, and transmits pain impulses throughout the brain [28].

Migraine with aura is associated with sensory, visual, or motor disturbances. These include increased sensitivity to light or sound, zig-zag flashes of light, and numbness or tingling in one hand or face. It is seen in about $30 \%$ of patients. Aura is considered to occur due to the slow wave of depolarization spreading across the cortex. This leads to stimulation of the trigeminovascular system, cerebral vessels nociceptors, and regions of the brain responsible for triggering pain and other neurological symptoms [29].

Studies with 31P-MRS have shown altered energy metabolism in the brain during acute migraine attacks. Hence, migraine can be triggered by fasting, physical exertion, excess sleep or sleep deprivation, intense aromas, or photosensitivity, which cause impaired oxygen metabolism due to mitochondrial dysfunction, oxidative stress, and inflammation [8,9]. Several oxidative stress biomarkers are found to have increased plasma levels in patients with migraine. Table 2 presents studies showing the association of migraine with inflammation and oxidative stress. 


\section{Cureus}

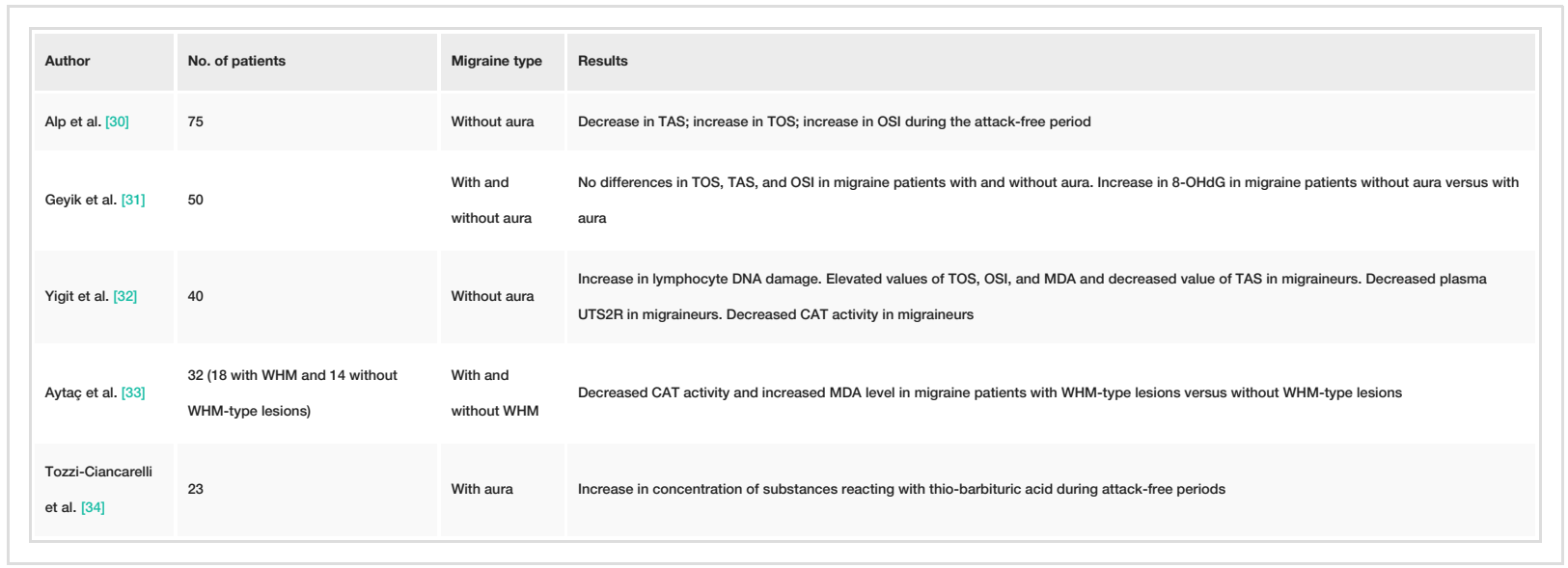

TABLE 2: Association of migraine with inflammation and oxidative stress.

TAS: total antioxidant status; TOS, total oxidant status; OSI: oxidative stress index; 8-OHdG: 8-hydroxydeoxyguanosine; MDA: malondialdehyde; UTS2R: urotensin 2 receptor; CAT: catalase; WHM: white matter hyperintensities

Due to their antioxidant properties and involvement in the mitochondrial electron transport chain, many nutraceuticals and herbal supplements such as coenzyme Q10, vitamin B2 (riboflavin), magnesium, alphalipoic acid, vitamin C, curcumin, and feverfew are considered for prophylaxis of migraine.

Coenzyme Q10, or ubiquinone, is a natural lipophilic substance needed for all cellular processes requiring energy. It acts as the complex III component of the electron transport chain and freely moves throughout the inner mitochondrial membrane transferring electrons from complex I and complex II (NADH dehydrogenase and succinate-Q-reductase, respectively) to cytochrome C. Coenzyme Q10 has been hypothesized to be useful in migraine prevention because of its vital role in sustaining mitochondrial energy stores [35]. In addition to its actions as an electron carrier, it also exerts an anti-inflammatory effect via nuclear factor kappa B signaling pathway inhibition preventing excessive reactive oxygen species production and inhibiting lipid membrane peroxidation and nucleic acid oxidation $[35,36]$. This helps with the inflammatory component of migraine. Recently, it is seen that coenzyme Q10 administered to migraine patients results in a decrease of CGRP, which is an important vasoactive peptide involved in the pathogenesis of migraine [13].

Curcumin has also been studied for the treatment of migraine. It is a lipophilic substance and acts as an anti-oxidant. It decreases the production of reactive oxygen species and inflammatory mediators such as interleukins (IL-1, IL-6) and cyclooxygenase [21].

Vitamin B2 (riboflavin) is a precursor for flavin-mononucleotide and flavin adenine-dinucleotide involved in the electron transport chain in the mitochondrial membrane. It is considered useful in migraine prevention due to its role in maintaining the energy stores of the body.

Magnesium (Mg) is involved in various biological processes. It acts as a cofactor for ATP-synthase which is involved in the production of ATP. Furthermore, it also regulates neuronal excitability and plays an important role in the regulation of vascular tone [37].

Feverfew (Tanacetum parthenium) is also thought to be helpful in migraine [38,39]. Since ancient days, it has been used for pain, inflammation, nausea, and vomiting [40]. It is largely found in South America. Its main active component is parthenolide which inhibits aldose reductase activity and causes relaxation of vascular smooth muscle. It also exhibits anti-inflammatory effects [41,42], all of which contribute to its anti-migraine effects.

The most common side-effects of magnesium and feverfew are limited to the gastrointestinal system and include nausea, diarrhea, appetite suppression, heartburn, and epigastric discomfort with an incidence of less than $1 \%[43,44]$.

Guilbot et al. conducted a prospective observational study on 132 adults, mainly women (91.2\%), in 2017 on the effect of a combination of coenzyme Q10, feverfew, and magnesium on the prevention of migraine. Supplementation significantly reduced the frequency of migraine attacks by the third month without any significant reduction in intensity. Overall, $75 \%$ of patients showed a reduction of at least $50 \%$ in the number of days with migraine headache per month [16].

Another study conducted by Gaul et al. in 2015 on the effect of coenzyme Q10, riboflavin, and magnesium 
supplementation on the clinical features of migraine included 130 adult migraineurs (age 18-65 years) with more than three migraine attacks per month. Patients were randomized in the treatment group and placebo group in a double-blinded manner and followed for three months. The frequency of headaches was reduced in the treatment group. The intensity of headache was also significantly reduced in the treatment group ( $\mathrm{p}=$ 0.03). The sum score of the HIT-6 (headache impact test) questionnaire was reduced in the treatment group [14].

Dahri et al. conducted a randomized, double-blind, placebo-controlled clinical trial in 2018 among 45 women aged 18-50 years to assess the effect of coenzyme Q10 on the clinical features and inflammatory markers of migraine. The treatment group received $400 \mathrm{mg} /$ day coenzyme Q10 for three months. Significant reduction in CGRP and TNF- $\alpha$ levels was seen $(\mathrm{p}=0.011$ and $\mathrm{p}=0.044$, respectively), but no significant difference in serum IL-6 and IL-10 was reported. A significant increase in coenzyme Q10 level $(\mathrm{P}<0.001)$ and reduction in the frequency, severity, and duration of migraine attacks was reported in the treatment group [13].

Two systematic reviews and meta-analyses on the effect of coenzyme Q10 in the prevention of migraine included 592 patients. Both studies concluded a significant reduction in the frequency of migraine attacks $(\mathrm{p}<0.0001)$ but no significant reduction in severity $[17,20]$.

A cohort study among 20 patients, aged 22-49 years, with a male/female ratio of 1:5 by Pucci et al. showed a significant reduction in the frequency of migraine attacks on supplementation of coenzyme Q10 [23].

Two randomized, double-blind, placebo-controlled clinical trials including 156 patients showed a significant reduction in the frequency, severity, and duration of migraine attacks $(\mathrm{p}<0.001)$ when coenzyme Q10 was supplemented with other supplements such as L-carnitine and nano-curcumin $[18,21]$.

Shoeibi et al. conducted an open-label, parallel, add-on, controlled trial on the effect of coenzyme Q10 in the prevention of migraine. A total of 80 patients were allocated to receiving only their current preventive drugs or their current preventive drugs plus $100 \mathrm{mg}$ coenzyme Q10 daily for three months. A significant reduction was evident in the frequency $(1.6$ vs. $0.5 ; \mathrm{p}<0.001)$ and severity of headaches $(2.3 \mathrm{vs.} 0.6 ; \mathrm{p}<$ 0.001) among coenzyme Q10 and control groups [15]. Other pilot studies also showed a reduction in the frequency of migraine attacks with coenzyme Q10 supplementation [19,22].

\section{Limitations}

We did not include any animal studies, case reports, and studies on the pediatric and adolescent populations. Moreover, we excluded studies published in other than the English language which may have impacted the study findings.

\section{Conclusions}

Studies discussed in this review confirm the involvement of oxidative stress in migraine, and therefore, antioxidants seem to be helpful in its prevention. Based on our research, several studies showed that vitamins and minerals including coenzyme Q10, magnesium, riboflavin, and feverfew reduced the number of days of migraine attacks in patients. Few studies also showed a decrease in the intensity and duration of migraine headaches. However, more research is needed to conclude the effects of herbal supplements on the prevention of migraine headaches. Because many migraine triggers are our day-to-day activities, physicians should educate their patients about lifestyle modification and suggest herbal supplements, vitamins, and minerals to patients who experience side effects from pharmaceutical drugs and desire a more natural treatment.

\section{Additional Information \\ Disclosures}

Conflicts of interest: In compliance with the ICMJE uniform disclosure form, all authors declare the following: Payment/services info: All authors have declared that no financial support was received from any organization for the submitted work. Financial relationships: All authors have declared that they have no financial relationships at present or within the previous three years with any organizations that might have an interest in the submitted work. Other relationships: All authors have declared that there are no other relationships or activities that could appear to have influenced the submitted work.

\section{References}

1. Butt JH, Franzmann U, Kruuse C: Endothelial function in migraine with aura - a systematic review . Headache. 2015, 55:35-54. 10.1111/head.12494

2. Global Burden of Disease Study 2013 Collaborators: Global, regional, and national incidence, prevalence, and years lived with disability for 301 acute and chronic diseases and injuries in 188 countries, 1990-2013: a systematic analysis for the Global Burden of Disease Study 2013. Lancet. 2015, 386:743-800. 10.1016/S01406736(15)60692-4 
3. Burch RC, Buse DC, Lipton RB: Migraine: epidemiology, burden, and comorbidity. Neurol Clin. 2019, 37:63149. 10.1016/j.ncl.2019.06.001

4. Burch RC, Loder S, Loder E, Smitherman TA: The prevalence and burden of migraine and severe headache in the United States: updated statistics from government health surveillance studies. Headache. 2015, 55:21-34. 10.1111/head.12482

5. Zaki EA, Freilinger T, Klopstock T, et al.: Two common mitochondrial DNA polymorphisms are highly associated with migraine headache and cyclic vomiting syndrome. Cephalalgia. 2009, 29:719-28. 10.1111/j.1468-2982.2008.01793.x

6. Eising E, de Vries B, Ferrari MD, Terwindt GM, van den Maagdenberg AM: Pearls and pitfalls in genetic studies of migraine. Cephalalgia. 2013, 33:614-25. 10.1177/0333102413484988

7. Eising E, Huisman SMH, Mahfouz A, et al.: Gene co-expression analysis identifies brain regions and cell types involved in migraine pathophysiology: a GWAS-based study using the Allen Human Brain Atlas. Hum Genet. 2016, 135:425-39. 10.1007/s00439-016-1638-x

8. Kabbouche MA, Powers SW, Vockell AL, LeCates SL, Hershey AD: Carnitine palmityltransferase II (CPT2) deficiency and migraine headache: two case reports. Headache. 2003, 43:490-5. 10.1046/j.15264610.2003.03095.x

9. Stuart S, Griffiths LR: A possible role for mitochondrial dysfunction in migraine . Mol Genet Genomics. 2012, 287:837-44. 10.1007/s00438-012-0723-7

10. Lateef TM, Cui L, Nelson KB, Nakamura EF, Merikangas KR: Physical comorbidity of migraine and other headaches in US adolescents. J Pediatr. 2012, 161:308-13. 10.1016/j.jpeds.2012.01.040

11. Pavlovic JM, Buse DC, Sollars CM, Haut S, Lipton RB: Trigger factors and premonitory features of migraine attacks: summary of studies. Headache. 2014, 54:1670-9. 10.1111/head.12468

12. Peroutka SJ: What turns on a migraine? A systematic review of migraine precipitating factors . Curr Pain Headache Rep. 2014, 18:454. 10.1007/s11916-014-0454-z

13. Dahri M, Tarighat-Esfanjani A, Asghari-Jafarabadi M, Hashemilar M: Oral coenzyme Q10 supplementation in patients with migraine: effects on clinical features and inflammatory markers. Nutr Neurosci. 2019, 22:607-15. 10.1080/1028415X.2017.1421039

14. Gaul C, Diener HC, Danesch U: Improvement of migraine symptoms with a proprietary supplement containing riboflavin, magnesium and Q10: a randomized, placebo-controlled, double-blind, multicenter trial. J Headache Pain. 2015, 16:516. 10.1186/s10194-015-0516-6

15. Shoeibi A, Olfati N, Soltani Sabi M, Salehi M, Mali S, Akbari Oryani M: Effectiveness of coenzyme Q10 in prophylactic treatment of migraine headache: an open-label, add-on, controlled trial. Acta Neurol Belg. 2017, 117:103-9. 10.1007/s13760-016-0697-z

16. Guilbot A, Bangratz M, Ait Abdellah S, Lucas C: A combination of coenzyme Q10, feverfew and magnesium for migraine prophylaxis: a prospective observational study. BMC Complement Altern Med. 2017, 17:433. 10.1186/s12906-017-1933-7

17. Parohan M, Sarraf P, Javanbakht MH, Ranji-Burachaloo S, Djalali M: Effect of coenzyme Q10 supplementation on clinical features of migraine: a systematic review and dose-response meta-analysis of randomized controlled trials. Nutr Neurosci. 2020, 23:868-75. 10.1080/1028415X.2019.1572940

18. Hajihashemi P, Askari G, Khorvash F, Reza Maracy M, Nourian M: The effects of concurrent coenzyme Q10, L-carnitine supplementation in migraine prophylaxis: a randomized, placebo-controlled, double-blind trial. Cephalalgia. 2019, 39:648-54. 10.1177/0333102418821661

19. Dalla Volta G, Carli D, Zavarise P, Ngonga G, Vollaro S: P026. Pilot study on the use of coenzyme Q10 in a group of patients with episodic migraine without aura. J Headache Pain. 2015, 16:A186. 10.1186/1129-237716-S1-A186

20. Sazali S, Badrin S, Norhayati MN, Idris NS: Coenzyme Q10 supplementation for prophylaxis in adult patients with migraine-a meta-analysis. BMJ Open. 2021, 11 :e039358. 10.1136/bmjopen-2020-039358

21. Parohan M, Sarraf P, Javanbakht MH, Foroushani AR, Ranji-Burachaloo S, Djalali M: The synergistic effects of nano-curcumin and coenzyme Q10 supplementation in migraine prophylaxis: a randomized, placebocontrolled, double-blind trial. Nutr Neurosci. 2021, 24:317-26. 10.1080/1028415X.2019.1627770

22. Vikelis M, Dermitzakis EV, Vlachos GS, et al.: Open label prospective experience of supplementation with a fixed combination of magnesium, vitamin B2, feverfew, Andrographis paniculata and coenzyme Q10 for episodic migraine prophylaxis. J Clin Med. 2020, 10:67. 10.3390/jcm10010067

23. Pucci E, Diamanti L, Cristina S, Antonaci F, Costa A: P032. Coenzyme Q-10 and migraine: a lovable relationship. The experience of a tertiary headache center. J Headache Pain. 2015, 16:A139. 10.1186/11292377-16-S1-A139

24. Ophoff RA, Terwindt GM, Vergouwe MN, et al.: Familial hemiplegic migraine and episodic ataxia type-2 are caused by mutations in the Ca2+ channel gene CACNL1A4. Cell. 1996, 87:543-52. 10.1016/s00928674(00)81373-2

25. De Fusco M, Marconi R, Silvestri L, et al.: Haploinsufficiency of ATP1A2 encoding the Na+/K+ pump alpha2 subunit associated with familial hemiplegic migraine type 2. Nat Genet. 2003, 33:192-6. 10.1038/ng1081

26. Asher JM, O'Hare L, Romei V, Hibbard PB: Typical lateral interactions, but increased contrast sensitivity, in migraine-with-aura. Vision (Basel). 2018, 2:7. 10.3390/vision2010007

27. Durham PL: CGRP-receptor antagonists--a fresh approach to migraine therapy? . N Engl J Med. 2004, 350:1073-5. 10.1056/NEJMp048016

28. D’Andrea G, Granella F, Cataldini M, Verdelli F, Balbi T: GABA and glutamate in migraine. J Headache Pain. 2001, 2:s57-60. 10.1007/s101940170011

29. Sutherland HG, Albury CL, Griffiths LR: Advances in genetics of migraine . J Headache Pain. 2019, 20:72. 10.1186/s10194-019-1017-9

30. Alp R, Selek S, Alp SI, Taşkin A, Koçyiğit A: Oxidative and antioxidative balance in patients of migraine . Eur Rev Med Pharmacol Sci. 2010, 14:877-82.

31. Geyik S, Altunısık E, Neyal AM, Taysi S: Oxidative stress and DNA damage in patients with migraine . J Headache Pain. 2016, 17:10. 10.1186/s10194-016-0606-0

32. Yigit M, Sogut O, Tataroglu Ö, et al.: Oxidative/antioxidative status, lymphocyte DNA damage, and 
urotensin-2 receptor level in patients with migraine attacks. Neuropsychiatr Dis Treat. 2018, 14:367-74. 10.2147/NDT.S156710

33. Aytaç B, Coşkun Ö, Alioğlu B, et al.: Decreased antioxidant status in migraine patients with brain white matter hyperintensities. Neurol Sci. 2014, 35:1925-9. 10.1007/s10072-014-1864-8

34. Tozzi-Ciancarelli MG, De Matteis G, Di Massimo C, Marini C, Ciancarelli I, Carolei A: Oxidative stress and platelet responsiveness in migraine. Cephalalgia. 1997, 17:580-4. 10.1046/j.1468-2982.1997.1705580.x

35. Pagano G, Aiello Talamanca A, Castello G, et al.: Current experience in testing mitochondrial nutrients in disorders featuring oxidative stress and mitochondrial dysfunction: rational design of chemoprevention trials. Int J Mol Sci. 2014, 15:20169-208. 10.3390/ijms151120169

36. Garrido-Maraver J, Cordero MD, Oropesa-Ávila M, et al.: Coenzyme q10 therapy. Mol Syndromol. 2014, 5:187-97. 10.1159/000360101

37. Daniel O, Mauskop A: Nutraceuticals in acute and prophylactic treatment of migraine . Curr Treat Options Neurol. 2016, 18:14. 10.1007/s11940-016-0398-1

38. Li R, Chen B, Wu W, Bao L, Li J, Qi R: Ginkgolide B suppresses intercellular adhesion molecule-1 expression via blocking nuclear factor-kappaB activation in human vascular endothelial cells stimulated by oxidized low-density lipoprotein. J Pharmacol Sci. 2009, 110:362-9. 10.1254/jphs.08275fp

39. Wu C, Chen F, Wang X, et al.: Antioxidant constituents in feverfew (Tanacetum parthenium) extract and their chromatographic quantification. Food Chem. 2006, 96:220-7. 10.1016/j.foodchem.2005.02.024

40. Pareek A, Suthar M, Rathore GS, Bansal V: Feverfew (Tanacetum parthenium L.): a systematic review . Pharmacogn Rev. 2011, 5:103-10. 10.4103/0973-7847.79105

41. Rajapakse T, Pringsheim T: Nutraceuticals in migraine: a summary of existing guidelines for use . Headache. 2016, 56:808-16. 10.1111/head.12789

42. Daniel O, Mauskop A: Nutraceuticals in acute and prophylactic treatment of migraine . Curr Treat Options Neurol. 2016, 18:14. 10.1007/s11940-016-0398-1

43. Sprenger T, Viana M, Tassorelli C: Current prophylactic medications for migraine and their potential mechanisms of action. Neurotherapeutics. 2018, 15:313-23. 10.1007/s13311-018-0621-8

44. Amir LH, Pirotta MV, Raval M: Breastfeeding--evidence based guidelines for the use of medicines . Aust Fam Physician. 2011, 40:684-90. 